\title{
La 'Teoría Crítica del Discurso' como herramienta metodológica para la investigación en teoría social
}

\author{
"Critical Theory of Discourse" as methodological tool for social theory inquiry
}

\author{
Eugenia Fraga \\ euge.fraga@hotmail.com \\ Facultad de Ciencias Sociales - Universidad de Buenos \\ Aires Instituto de Investigaciones Gino Germani, \\ Argentina
}

Recepción: 31 Marzo 2020

Aprobación: 08 Noviembre 2020

Publicación: 01 Diciembre 2020

Cita sugerida: Fraga, E. (2020). La 'Teoría Crítica del Discurso' como herramienta metodológica para la investigación en teoría social. Cuestiones de Sociología, 23, e107. https://doi.org/10.24215/23468904e107

\begin{abstract}
Resumen: En este trabajo daremos forma a una metodología particular dentro del trabajo en teoría social, llamada "Teoría Crítica del Discurso". Esta metodología se inscribe dentro de la discusión abierta en los últimos años acerca de los modos y técnicas de trabajo teórico en las ciencias sociales, y resulta de una combinación entre la Teoría Crítica y el Análisis Discursivo. Se estudiará especialmente el antecedente de la corriente conocida como Análisis Crítico del Discurso o ACD, de Norman Fairclough y Ruth Wodak, y se pasará revista a los aportes de autores fundamentales de la teoría social crítica a las reflexiones en torno al lenguaje y el discurso, como ser Max Horkheimer, Charles Wright Mills, Herbert Marcuse, Alvin Gouldner y Jürgen Habermas.
\end{abstract}

Palabras clave: Teoría crítica, Teoría social, Metodología de la teoría, Análisis discursivo, Teoría crítica del discurso.

\begin{abstract}
In this work we will give shape to a particular methodology for social theory inquiry called "Critical Theory of Discourse". This methodology inscribes itself in the recently opened discussion around the modes and techniques of theoretical research in the social sciences, and it results from a combination between Critical Theory and Discourse Analysis. We will study most specially the antecedent of Critical Discourse Analysis or CDA by Norman Fairclough and Ruth Wodak, and we will go through the contributions of key authors of critical social theory such as Max Horkheimer, Charles Wright Mills, Herbert Marcuse, Alvin Gouldner and Jürgen Habermas.
\end{abstract}

Keywords: Critical theory, Social theory, Methodology of theory, Discourse analysis, Critical theory of discourse.

\section{Introducción: la Escuela de Frankfurt, la Nueva Izquierda y la Metodología de LA TEORÍA}

La propuesta que aquí presento se enmarca en lo que he venido trabajando a lo largo aproximadamente de una década, y por ello constituye en parte un punto de llegada, aunque también se trata de una propuesta que deberá seguir desarrollándose en los años venideros, pues aquí se presentan apenas sus líneas más generales. Entonces, este artículo se inscribe en una línea de investigación de largo plazo, que contó desde el principio con dos objetivos. Por un lado, desde 2014 tengo el propósito de 1) construir una historia conceptual de la teoría crítica, esto es, una historia de las conceptualizaciones que distintas modalidades de 
la teoría crítica han producido, en el marco de dos tradiciones de pensamiento diferentes pero con muchos puntos de contacto: la Escuela de Frankfurt, de origen alemán, y la Nueva Izquierda de cuño estadounidense. En este sentido, desde mi tesis de maestría (2014-2016), pasando por mi tesis de doctorado (2017-2018), y llegando hasta la investigación postdoctoral actualmente en curso (2019-2020), he ido deteniéndome -y tengo la intención de seguir haciéndolo- en distintas "estaciones" de dicha historización conceptual.

Por otro lado, mi otro objetivo de largo plazo (desde 2013) es 2) realizar una autorreflexión epistemológicametodológica acerca de la investigación en teoría social. En otras palabras, desde mis primeras experiencias de investigación como estudiante de grado, pasando por las dos tesis, y nuevamente aquí, en este primer producto de mi investigación postdoctoral, me ha interesado indagar en las posibilidades y limitaciones, así como en ciertas modalidades especificas - esto es, críticas- de teorización social. En efecto, y en el marco del equipo de investigación en el que participo desde 2011 (el Grupo de Estudios sobre Problemas y Conceptos de la Teoría Social, del Instituto de Investigaciones Gino Germani, Buenos Aires, Argentina) he trabajado desde 2013 en torno a la epistemología decolonial/poscolonial (ver por ejemplo Fraga, 2015b). Luego, he podido reflexionar desde 2015 acerca de la producción teórica en general (ver por ejemplo Fraga, 2018a) y en el contexto latinoamericano en particular (ver por ejemplo Fraga, 2018b), al igual que algunos de mis compañeros (Álvarez Ruiz, 2019; Sasín, 2019; Trovero, 2019).

Ahora bien, como había adelantado, estos dos objetivos sobrepasan el presente escrito; sin embargo, puedo decir algo sobre ellos a continuación. En cuanto al primer objetivo: si en la tesis de maestría realicé un análisis histórico-conceptual de la teoría crítica de Max Horkheimer (Fraga, 2017), y en la tesis doctoral hice lo propio con Charles Wright Mills y Herbert Marcuse (Fraga, 2019a), en la investigación postdoctoral realicé el análisis histórico-conceptual de la teoría crítica de Alvin Gouldner y Jürgen Habermas. Esto último es lo que retomaré un poco más abajo para luego, en función de ello, comenzar a sistematizar una "teoría crítica de los discursos", que es, en mi opinión, la modalidad específica de la teoría crítica desplegada por estos cinco autores.

Finalmente, en función de los lineamientos generales que esbozaré de esta teoría crítica de los discursos, sistematizada a partir de los aportes conjugados de los cinco autores, bosquejaré a su vez los primeros cimientos de una herramienta metodológica que creo podría ser de provecho para la investigación teóricosocial actual. Pues así como la investigación empírica en las ciencias sociales cuenta con una rica diversidad de metodologías (como la etnografía, la observación participante, la descripción densa, la entrevista en profundidad, la historia de vida, el análisis documental, la encuesta, la codificación, el análisis estadístico, etc.), es más difícil afirmar lo mismo acerca de la investigación teórica. No por falta de interesantes e importantes investigaciones teóricas, sino porque no se encuentra igualmente explicitado e institucionalizado el herramental metodológico.

Es cierto que en las últimas décadas (desde los noventas del pasado siglo) ha surgido un debate en torno a lo que podemos llamar la "metodología de la teoría”, esto es, de la investigación en teoría social. De tal modo, pueden nombrarse algunos abordajes, emergidos en los países centrales, como el de la Metateoría (Ritzer, 1990), la Historia Intelectual (Skinner, 2000), la Historia Conceptual (Koselleck, 2012), la Metaforología (Blumenberg, 2003), la Problematización (Castel, 2007), la Geografía del Saber (Pels, 2001), la Sociología Histórica de la Formación de Conceptos (Somers, 1995) y la Historia de la Sociología con Propósito Sistemático (Schluchter, 2008; hemos hecho un recuento y una evaluación comparativa de estas diversas perspectivas en Fraga, 2019b).

En nuestro propio ámbito local, la Argentina, también existen una serie de colegas que han aportado en los últimos años (desde el cambio de siglo, y sobre todo en la última década) a esta discusión desde la sociología y otras ciencias sociales, como Aguilar, Glozman, Grondona y Haidar (2014) con la Construcción de Corpus; Alvaro (2017) con la Deconstrucción de la Sociología; Bialakowsky (2013) con el Abordaje Problemático; Cristiano, Torres, Gonnet y Abril (2015) con las 7 Operaciones y Procedimientos de Construcción Teórica, 
de Marinis (2013) con el Destilado de Registros; Palti (2005) con la Historia de los Lenguajes Políticos; y Pignuoli Ocampo (2013) con la Teoría Sociológica Comparada. En cuanto a los colegas latinoamericanos más destacados en el marco de la discusión metodológica sobre la teoría social, remitimos a Abend, 2008; Girola, 2011; Mascareño, 2006; Requena Santos, 2000; y Zabludovsky, 2002. Justamente, entonces, con este escrito me gustaría intervenir en el seno de estas discusiones actuales, y considero que es factible hacerlo dando forma a una herramienta metodológica específica: la "Teoría Crítica del Discurso", inspirada en aquella variante crítica de la teoría social mencionada más arriba y que, a continuación, me encargaré de detallar.

\section{Hipótesis: Hermenéutica, Pragmatismo, Giro lingüístico, Marxismo culturalista, ANÁLISIS DISCURSIVO}

Parto aquí de tres hipótesis de investigación. En primer lugar, sostengo que el desarrollo de una historia conceptual de la teoría crítica da lugar a la construcción de una concepción alternativa de teoría crítica, esto es, una que incluye a la tradición de la Escuela de Frankfurt alemana, con la que suele igualarse -Horkheimer, Marcuse, Habermas- pero que combina a esta con la tradición de la Nueva Izquierda estadounidense de sociología -Wright Mills, Marcuse, Gouldner-. ¿Por qué recurrir a esta serie de autores? Su vinculación con la teoría crítica es prácticamente obvia en el caso de Horkheimer -fundador y organizador de la Escuela de Frankfurt- y también en el de Habermas -figura más renombrada de la segunda generación de dicha Escuela-. El caso de Marcuse es menos obvio pero igualmente fácil de defender - pues sí estuvo durante varios años vinculado a la Escuela de Frankfurt, aunque luego desarrolló una perspectiva con vetas singulares que lo acercó a la Nueva Izquierda-. Son los casos de Wright Mills y Gouldner los que más sorprenden a quienes escuchan la propuesta, pues no pertenecieron a la institucionalidad de dicha Escuela.

Sin embargo, no sólo es claro al leer sus obras que ambos autores explicitan constantemente su opción por el despliegue y la defensa de una teoría crítica en las ciencias sociales y humanas, sino que además varias de sus referencias teóricas a lo largo de sus textos son vínculos directos con los pensadores frankfurtianos, con los cuales además compartieron espacios de trabajo en Estados Unidos mientras los frankfurtianos, provenientes de Alemania, estaban allí exiliados. Aún más, como se verá a continuación, existen continuidades y afinidades teóricas entre ambos conjuntos de perspectivas. Así, por sólo adelantar algunos tópicos, existen amplias coincidencias entre los análisis críticos de los medios de comunicación de masas y la industria cultural realizados por Horkheimer, Marcuse y Wright Mills, del mismo modo que existen fuertes superposiciones entre la propuesta de Habermas de la racionalidad comunicativa del discurso y la propuesta de Gouldner de una cultura del discurso crítico.

Esta combinación de autores, por otra parte, proviene de maridar el marxismo heterodoxo de ambas tradiciones -la frankfurtiana y la nuevaizquierdista-, con la hermenéutica - por ejemplo, de Hans Georg Gadamer (1997) o Martin Heidegger (2012), en el caso de los diversos autores de la Escuela de Frankfurtpero también con el pragmatismo - por ejemplo, de Charles Sanders Peirce (1934) y George Herbert Mead (1934), en el caso de la Nueva Izquierda, pero también de Habermas-. Por supuesto, tanto la hermenéutica como el pragmatismo son corrientes fuertemente concentradas en el estudio del lenguaje y la significación, con lo cual resultan lógicos sus "aportes subterráneos" a una "Teoría Crítica del Discurso" como la que quiero formular aquí. Es que, de manera incipiente y latente en Horkheimer, Marcuse y Wright Mills, y de manera consciente y explícita en Habermas y Gouldner, su teoría crítica resulta anclarse crecientemente en aquella revolución, dentro del pensamiento occidental, conocida como "giro lingüístico": el cambio de foco de la conciencia al lenguaje como factor explicativo principal de la especificidad humana.

En segundo lugar, sostengo la hipótesis de que las modalidades particulares de teoría crítica desplegadas por Horkheimer, Wright Mills, Marcuse, Gouldner y Habermas, efectivamente permiten dar forma a una Teoría Crítica de los Discursos. Esta idea nos permite avanzar aún un paso más respecto a la hipótesis anterior. No se trata entonces solamente de que exista entre los diversos autores una coincidencia en cuanto a su 
posicionamiento crítico frente a la sociedad y en la teoría, sino que todos ellos convergen en torno a un tema de consecuencias fundamentales: el lenguaje, la comunicación, el discurso. Esto es lo que hace a las formulaciones de estos cinco pensadores, en nuestra opinión, merecedoras de alimentar la propuesta teóricometodológica que estamos presentando.

En el caso de los autores más viejos -Horkheimer, Marcuse, Wright Mills-, se trata de algunos de los herederos más importantes a nivel internacional de los antiguos abordajes interesados en dicha dimensión de lo social. Me refiero, en el caso de Wright Mills, al pragmatismo -enfocado en los problemas de la argumentación y la lógica-; y, en los casos de Horkheimer y Marcuse, a la hermenéutica -abocada a los problemas de la interpretación y la comprensión-. Pero además, estos autores se nutren todos ellos del llamado "marxismo culturalista" (Gyorgy Lukács, 2013; Karl Korsch, 1971; Antonio Gramsci, 1984; entre otros) -escuela particular dentro del marxismo, centrada en el estudio de los símbolos y las representaciones, por ejemplo, típicamente, de la ideología-. En el caso de los autores más nuevos -Gouldner y Habermas-, ellos no sólo cargan ambos con la misma triple herencia del pragmatismo, la hermenéutica y el marxismo culturalista, sino que además, de forma novedosa, se inscriben clara y explícitamente dentro del giro lingüístico, por lo que el lenguaje, la comunicación y el discurso devienen el centro mismo de todas sus preocupaciones, como se verá más adelante.

Entonces, este escrito busca concentrarse en las afinidades, continuidades y convergencias entre los cinco autores. Por supuesto que existen singularidades que hacen a cada una de sus propuestas diferentes y distinguibles entre sí, e incluso han habido discusiones teórico-epistemológicas entre sus representantes. Por ejemplo, podemos mencionar cierta distancia entre el pesimismo de Horkheimer frente a una mirada más esperanzada en el caso de Marcuse y Wright Mills, o también el señalamiento por parte de los autores posteriores, Habermas y Gouldner, acerca de un insuficiente corrimiento, por parte de los demás, respecto de una filosofía de la conciencia. Sin embargo, no es el objeto de este trabajo analizar dichas discrepancias, las cuales por otro lado han sido extensamente elaboradas por parte de otros investigadores.

En este sentido, no concordamos con quienes consideran que las diferencias entre las distintas perspectivas sean lo suficientemente profundas como para impedir el diálogo entre ellas, y mucho menos concordamos con que intentar ponerlas en diálogo redunde en propuestas "eclécticas". En efecto, las semejanzas entre estas cinco perspectivas superan a las diferencias, por lo que de ningún modo se trata de paradigmas incompatibles ni inconmensurables. Y por otra parte, es precisamente del trabajo que compara y complementa, que une y adiciona, que el conocimiento sobre lo humano y lo social logra adquirir un punto de mira más complejo, mejor capacitado para comprender, explicar -y eventualmente transformar- un mundo con infinitos matices.

Finalmente, en tercer lugar, sostengo que la Teoría Crítica de los Discursos puede constituir una herramienta metodológica fructífera para la investigación teórico-social, especialmente de tipo crítico. La Teoría Crítica del Discurso es en este sentido un abordaje similar pero distinguible respecto del Análisis Discursivo clásico. Por empezar, si la primera se nutre de tradiciones germanas y anglosajonas, el segundo lo hace en cambio de tradiciones principalmente francesas -desde Ferdinand de Saussure (1997) hasta Oswald Ducrot (1990) o Michel Pêcheux (2016), por sólo nombrar unos pocos referentes-. Además, mientras la Teoría Crítica del Discurso ha sido utilizada por los propios Horkheimer, Wright Mills, Marcuse, Gouldner y Habermas -aunque sin llamarla de ese modo-, en la investigación teórico-conceptual el Análisis Discursivo clásico ha sido usado en cambio sobre todo en el estudio de textualidades propias de la investigación históricodocumental -desde el discurso mediático hasta el discurso político-.

Esta es una de las principales razones por las que, para las ciencias sociales, no podemos contentarnos con el análisis discursivo clásico, sino que precisamos herramientas más específicas para nuestros puntos de vista. Sin embargo, cabe destacar que, dentro del propio Análisis Discursivo, existe una variante no tan conocida aún que sí se acerca más a nuestros intereses, el llamado "Análisis Crítico del Discurso" o ACD, variante en la cual ahondaremos más adelante para marcar semejanzas y diferencias con la Teoría Crítica de 
los Discursos. Yo misma (Fraga, 2014) realicé un análisis discursivo; y vinculé el análisis discursivo a la teoría crítica frankfurtiana (Fraga, 2015a). Así también, algunas colegas han utilizado el análisis discursivo desde las ciencias sociales (Montero, 2012; Fernández Cordero, 2013), o con intención crítica (Labandeira, 2008).

A continuación, sistematizaré los que considero aportes clave de las propuestas de Horkheimer, Wright Mills, Marcuse, Gouldner y Habermas a una “Teoría Crítica de los Discursos”. Lo haré combinando por un lado los resultados de mis propias investigaciones histórico-conceptuales anteriores, junto con los análisis que diversos comentaristas sobre sus respectivas obras han hecho al respecto de tal posibilidad. Veamos entonces cuáles son esos aportes siguiendo un despliegue cronológico asociado a lo que pueden concebirse como las tres primeras "estaciones" de una "teoría crítica alternativa" -aquella que combina elementos "frankfurtianos" y "nuevaizquierdistas"-. La primera estación es la de Horkheimer -y también la de Theodor Adorno (2008)-, quienes desarrollan sus reflexiones aproximadamente entre 1920-1970. La segunda estación es la de Wright Mills y Marcuse, que escriben entre 1930-1980 -aunque Wright Mills muere tempranamente-. La tercera estación -y la última que aquí abarcamos- es la de Gouldner y Habermas, cuya obra se despliega entre 1950-2010. Además de estas tres, hay una cuarta estación y una quinta y actual de esta teoría crítica alternativa o ampliada, conformadas por autores alemanes y estadounidenses como Axel Honneth (1997) y Nancy Fraser (1997) por un lado (1970-actualidad), y Hartmut Rosa (2015) y Rahel Jaeggi (2018) por el otro (1990-actualidad). Sus respectivos aportes potenciales a una Teoría Crítica del Discurso quedan para futuras investigaciones que emprenda.

\section{Propuesta: la Teoría Crítica de los Discursos de Horkheimer, Wright Mills, Marcuse, Gouldner y Habermas}

Ahora sí, podemos pasar a desplegar los lineamientos conceptuales generales de una teoría crítica del discurso que se nutra de las cinco perspectivas mencionadas. Cabe aclarar en este punto que lo que haré a continuación no será un análisis crítico de estos autores, de sus tradiciones ni de sus conceptos, es decir, no se tratará de una evaluación de sus posibles dificultades o limitaciones -como por otra parte toda teoría presenta-, pues lo que me interesa es lo contrario: destilar la máxima cantidad posible de herramientas conceptuales para que luego puedan ser utilizadas en el análisis discursivo de textos sociales. Tampoco se tratará, a continuación, de un análisis social de los autores, tradiciones y conceptos, es decir, no me encargaré de poner a los textos en sus contextos geopolíticos, epocales ni académicos -tarea típica de una Historia Intelectual-, puesto no sólo que existen las obvias limitaciones de espacio de cualquier escrito, que obligan a diversos recortes en la tarea a emprender, sino que como ya he mencionado, mi objetivo es otro. Mi objetivo es estrictamente conceptual, es decir que mi intención a continuación será mencionar una serie de conceptos, extraídos de las obras de los distintos autores, que considero pueden resultar productivos para posteriores análisis discursivos de carácter crítico.

Además, al presentar dichas series de conceptos en función de un despliegue mayormente cronológico de autores, podrán irse apreciando las sutiles transformaciones en las reflexiones de la teoría crítica acerca del lenguaje, la comunicación y el discurso. Por ejemplo, si en los escritos de la década del treinta aparece un mayor énfasis en temas clásicos del marxismo, como el del discurso ideológico, en los escritos de la década del cincuenta priman los estudios de los medios de comunicación, y a partir de la década del ochenta dominan las propuestas fundamentales de una filosofía del lenguaje. Esta atención a las transformaciones en las conceptualizaciones es algo típico de una perspectiva como la Historia Conceptual. Otra aclaración importante es el hecho de que, como se verá, he destilado los conceptos no sólo de las obras más renombradas de los diversos autores, sino que me he adentrado también en textos generalmente considerados "menores", desde diarios de juventud hasta cartas y otros escritos autobiográficos, puesto que, en mi opinión, es muchas veces allí donde se encuentran las propuestas conceptuales más aventuradas, las cuales frecuentemente son pasadas por alto. Hechas estas observaciones, ahora sí comenzaré el destilado conceptual. 
En el caso de la obra de Max Horkheimer pueden encontrarse cinco elementos para una Teoría Crítica del Discurso. a) Desde sus escritos tempranos, como el diario Ocaso, puede hallarse una crítica del "lenguaje especializado" o discurso científico, por su aislamiento de la dimensión moral respecto de lo cognitivo (Horkheimer, 2002, pp. 57-59). b) Especialmente en sus primeros ensayos académicos, como los reunidos en Historia, metafísica y escepticismo, hay una crítica del discurso ideológico, que muestra su "función social" y cuestiona sus "sentidos dados" (Horkheimer, 1995, p. 78).c) En sus ensayos clásicos de la etapa frankfurtiana, publicados en Teoría crítica, se observa una crítica del discurso de sentido común, cuyo "poder integrador" lo vuelve incapaz de generar significados novedosos (Horkheimer, 1974, p. 187). d) En el famoso y coautorado libro Dialéctica de la ilustración puede verse una crítica del discurso ilustrado occidental más en general, que reemplaza los "conceptos dialécticos" por meras "fórmulas repetitivas o funcionales" (Horkheimer y Adorno, 2001, p. 140). e) Frente a todo lo anterior, en Critica de la razón instrumental, su libro más sistemático, el autor propone finalmente pulir un lenguaje "orientado a la verdad" y con capacidad crítica, vinculado con las "estructuras generales de la racionalidad" humana. Basado en esa razón, es preciso un "esfuerzo idiomático" que llame "a las cosas por su verdadero nombre" y en función del cual mostrar las contradicciones veladas en y por la sociedad existente. Para lograr el cometido, ese discurso develador no puede formarse con "frases aisladas" sino que debe constituir una "teoría sistemática" (Horkheimer, 1973, p. 187).

A pesar de todos estos ricos aportes de Horkheimer a la cuestión lingüística, sin embargo, la literatura secundaria no los ha señalado lo suficiente. Las únicas excepciones son Crook (1991) -que subraya la crítica horkheimeriana al lenguaje integrador-y Schmidt (1998) -que resalta la distinción horkheimeriana entre palabra y retórica-. Algo similar sucede con W right Mills, donde tampoco en este caso los comentaristas han notado suficientemente el aporte de este autor al estudio del lenguaje. Las dos excepciones son Horowitz (1970) -que remarca el estilo discursivo wrightmillsiano con su combinación de cientificismo y periodismoy Loy y Booth (2004) -quienes observan el lugar de herramienta que Wright Mills otorga al lenguaje-.

En nuestra opinión, en la obra de Charles Wright Mills pueden encontrarse siete elementos útiles para una Teoría Crítica del Discurso. a) Ya en su tesis doctoral, Sociología y pragmatismo, hay una crítica de la actitud y la epistemología utilitaristas, que lingüísticamente definen la "verdad" de todo enunciado a partir de su "éxito" práctico (Wright Mills, 1968, p. 235). b) En distintos ensayos de los cuarentas y los cincuentas, como los reunidos en De hombres sociales y movimientos políticos, puede verse una crítica del "vocabulario común" distorsionado por los medios de comunicación de masas, producido en la dinámica del "aparato cultural" dominante (Wright Mills, 1970, pp. 495-496). c) En su libro Los marxistas asimismo puede observarse una crítica del proceso corriente de trastrocamiento de "ideales" en "ideologías", es decir, en "retóricas vacías" respecto de su anterior contenido tanto normativo como cognitivo, especialmente en los casos del discurso político y del discurso científico (Wright Mills, 1976, p. 81). d) En el clásico manual de psicología social coautorado, Carácter y estructura social, puede hallarse un análisis de corte más general acerca de todo discurso en tanto constituido, por un lado, por "símbolos maestros" o de justificación, y por otro, por "contrasímbolos" de protesta, así como de su interrelación y su vínculo con el cambio social (Wright Mills y Gerth, 1961, pp. 274-289). e) Esto se apoya en su temprana propuesta, en los escritos compilados bajo el título Poder, política y pueblo, de un análisis "sociótico" -como distintivo respecto del análisis semiótico- de la relación entre lógica y cultura, que pueda señalar la presencia de "creencias acríticas" incluso en el discurso científico (Wright Mills, 1963, p. 492). f) En su obra más conocida, La imaginación sociológica, se realiza en esa misma línea un análisis sintáctico, semántico y pragmático del lenguaje, especialmente del discurso sociológico y de sus variantes dominantes, la "gran teoría” y el "empirismo abstracto" (Wright Mills, 1961, pp. 52-91).g) Frente a todo lo anterior, finalmente, el autor propone en sus Cartas y escritos autobiográficos un modo de trabajo "lúdico" y "artesanal" con las palabras, al que titula "poesía sociológica” (Wright Mills, 2000, pp. 112-113).

En cuanto a la obra de Herbert Marcuse, pueden encontrarse cinco elementos para una teoría crítica del discurso. a) En el libro El marxismo soviético, hay un análisis del proceso por el cual discursos originariamente críticos pueden devenir dogmáticos -ejemplo del "discurso oficial" soviético- (Marcuse, 
1984, pp. 139-142). b) En su obra más famosa, El hombre unidimensional, puede verse un análisis del proceso por el cual el "lenguaje bidimensional y dialéctico" que dio origen a la modernidad acabó deviniendo "lenguaje unidimensional y cerrado" -ejemplo del discurso dominante en el mundo capitalista- (Marcuse, 1985, pp. 127-137). c) En Contrarrevolución y revuelta, se observa un análisis del proceso por el cual los discursos "contraculturales" acaban siendo cooptados por los discursos dominantes, gracias a factores como su "antiteoricismo" y su conversión en "vocabularios fetichizados" (Marcuse, 1972, pp. 29 y 85). d) Asimismo, puede observarse una crítica de cómo todo lo anterior impacta en el discurso científico, con sus conceptos "operacionales, funcionales y conductistas" (Marcuse, 1985, pp. 45-47; 136-138; 170 y 199). e) Frente a todo esto, finalmente, en La dimensión estética el autor apuesta por un discurso crítico que ancle en su propia "formalización estética", es decir, que busque su modelo en el "lenguaje del arte", por un lado, y en la "dimensión sensible" del ser humano, por otro (Marcuse, 1978, pp. 142-144).

A pesar de toda esta riqueza, tampoco en este caso la bibliografía secundaria ha hecho suficientes aclaraciones respecto a los aportes de Marcuse a la dimensión lingüística de lo social. Como excepciones aisladas están Benhabib (1986) -quien subraya el foco marcusiano en las experiencias psicológicas prelingüísticas- y Slater (1975) -quien analiza la crítica marcusiana del lenguaje político unidimensional-. Y una vez más, lo mismo sucede en el caso de Gouldner, en el que la literatura no ha enfatizado lo suficiente sus contribuciones sociolingüísticas. Las excepciones son Aune (1990) -quien muestra cómo el concepto gouldneriano de cultura del discurso crítico o CDC tuvo influencia en el lenguaje académico de finales del siglo pasado-, y Daley (1991) -quien resalta los estudios gouldnerianos sobre la esfera pública y los medios de comunicación de masas-.

Sin embargo, sobre la base de mis indagaciones sobre las obras de Alvin Gouldner, veo seis potenciales aportes suyos para una Teoría Crítica de los Discursos. En La dialéctica de la ideología y la tecnología, aparecen dos puntos relevantes para la cuestión que tenemos entre manos: a) un análisis teórico del nivel "paleosimbólico" de los discursos, esto es, de lo "prelingüístico" y "presimbólico", como distinto del nivel lingüístico y simbólico propiamente dicho, y (Gouldner, 1978, pp. 269-270); y b) un análisis histórico de la "revolución comunicacional" y sus efectos en los "discursos ideológicos" y su "gramática", acompañada de una propuesta de "política crítica de los medios informativos" (Gouldner, 1978, p. 156). c) En el reconocido La crisis de la sociología occidental, hallamos un análisis de los "supuestos básicos subyacentes" de toda teoría, o también, de los enunciados implícitos que conforman la "subestructura" de los enunciados explícitos superestructurales. La hipótesis aquí es que el tono de cada nueva teorización viene dado por su concepción de la lengua, por ejemplo, en términos de "íconos" y "códigos" (Gouldner, 1973, pp. 34-63). d) Aún con mayor especificidad, en La sociología actual: renovación y crítica, aparece un análisis crítico del discurso científico-social, en sus variantes tanto "objetivistas" como "partidarias", así como en función de sus herencias tanto "románticas" como "clasicistas", y en su tono "trágico" generalizado (Gouldner, 1979, pp. 29 y 310-317). e) En paralelo, en Los dos marxismos hay un análisis del lenguaje marxista en particular, desarrollado a partir de la premisa de "tomarse el lenguaje en serio": esto supone elaborar en los "estilos cognitivos", "estructuras formales", "sistemas de explicación”, "autorías ficcionales" y "anomalías" del discurso teórico y político (Gouldner, 1983, p. 35). f) Como corolario de todo lo anterior, el autor construye, en El futuro de los intelectuales y el ascenso de la nueva clase, una teoría de la "clase intelectual" en tanto "comunidad de habla", asociada a lo que denomina su "cultura del discurso crítico". Allí se despliegan sus aserciones, justificaciones, autoridades, argumentaciones, refutaciones, "tecnicalidades", reflexividades, disciplinas, renuncias, explicitaciones, contextualizaciones, sensibilidades, etc. (Gouldner, 1980, p. 48). Como afirma en En contra de la fragmentación, sistematizar tal teoría de la CDC propia de los intelectuales incluye también el análisis de sus "binarismos", "paradojas", "confrontaciones", "metaforicalidades" y "paleosimbolismos". En otras palabras, se trata de conectar los discursos con sus dimensiones sociales, históricas, políticas, pero también con sus dimensiones creativas y subjetivas (Gouldner, 1985, pp. 64-70; 223-239 y 279). 
Finalmente, sobre la base de mis indagaciones sobre las obras de Jürgen Habermas y el relevamiento de bibliografía secundaria, puedo al fin decir que sus posibles aportes a una teoría crítica de los discursos sí son más conocidos, aunque aún poco sistematizados para el ámbito de la teoría social -como distinta a una teoría jurídica, en donde sí me preexiste una mayor sistematización- (ver Alvear, 2015; Barbieri Durao, 2015; Brígido, 2017; Casagrande y Casagrande, 2011; Fernández, 1997; Gil Martín, 2005; Herrera Gómez, 2003; Lubenow, 2011; Persch, 2009; Repa, 2008; Soares Teixeira, 2016; Torres Oviedo, Galván Patrignani y Hernández Palma, 2016). En particular, Cukier, Bauer y Middleton (2003), Diniz Focas (2016) y Palma (2011) han sugerido la productividad de la propuesta habermasiana para el estudio del discurso y el lenguaje de manera explícita, especialmente en lo que hace a su concepto de "pretensiones de validez", y a su fructífero entrecruzamiento con analistas del discurso como Ducrot y filósofos del lenguaje como Gadamer.

Junto a ellos, y más allá de ellos, entreveo siete posibles aportes de Habermas para una teoría crítica del discurso. a) Ya en Historia y crítica de la opinión pública encontramos un análisis del surgimiento histórico de la "esfera pública", que pone especial atención a la publicidad literaria en relación con la publicidad política. Se distingue entre "esfera pública”, "esfera privada” y "esfera íntima”, así como entre "publicidad”, "esfera pública" y "opinión pública". Y se indaga en las formas periodísticas y de "comunicación de masas" (Habermas, 1981, pp. 215; 227 y 272). b) En El discurso filosófico de la modernidad se despliega un debate entre el habla y la escritura como fenómenos centrales para la comprensión de la sociedad moderna, así como una discusión acerca de la problemática de los "géneros discursivos" (Habermas, 2008, pp. 161-184). c) En La lógica de las ciencias sociales hay un análisis de la tradición "hermenéutica" de la interpretación de textos. Se indaga en su lógica, su dualismo, su metodología, su actualidad, su universalismo, y su pretensión de "comprensión del sentido" (Habermas, 1987, pp. 79-306). Respecto de lo anterior, en Conocimiento e interés se profundiza especialmente en su variante "historicista"; se analizan sus ideas de la "comprensión de la expresión", la "comunicación lingüística" y la "crítica del significado" (Habermas, 1982, pp. 147-157 y 179-180). Asimismo y dentro del ámbito sociológico en particular, en Estudios preliminares a la teoría de la accióncomunicativa se rastrean los "fundamentos lingüísticos" de la fenomenología social: sus "pretensiones de validez" y su "monadología” (Habermas, 2001, pp. 23-44).d) Pero además de la tradición hermenéutica se analiza también la tradición "pragmática" y su "filosofía del lenguaje"; se estudian sus "lógicas de lenguaje", su "realismo de los universales" y sus propias "críticas del significado" (Habermas, 1982, pp. 101-115). Así, y en el ámbito sociológico en particular, se rastrean los "fundamentos lingüísticos" del pragmatismo, especialmente sus "usos comunicativos y cognitivos del lenguaje" (Habermas, 2001, pp. 45-104). e) En función de los análisis anteriores, el autor presenta una propuesta de "pragmática universal" basada en una "teoría de la verdad" particular, con nociones como "interacción lingüística" y "patología comunicativa" (Habermas, 2001, pp. 129-169). En Sobre la pragmática comunicativa se vincula esta propuesta a las diversas formas de la "racionalidad comunicativa" y a las "teorías críticas del sentido", con foco en nociones como "actos de habla", "mediaciones lingüísticas”, etc. Allí destaca asimismo la distinción entre "usos poéticos” y "comunicativos" del lenguaje (Habermas, 1998, pp. 183-256; 383 y 401).

Este proyecto habermasiano se sistematiza, en la famosa gran obra de dos tomos Teoría de la acción comunicativa, a partir de conceptos como "susceptibilidad de crítica de afirmaciones", "espectro de manifestaciones y emisiones", "proceso, procedimiento y producto de la argumentación", "formas y campos argumentales", “comprensión mítica y desencantada del mundo”, "reflexividad comunicativa”, "lenguaje orientado al éxito o al entendimiento", "efectos perlocucionarios e ilocucionarios" y "significado literal y contextual". Asimismo, se estudian el "lenguaje subhumano de gestos", la "interacción simbólicamente mediada", el "trasfondo normativo de la comunicación", su "componente expresivo", la "lingüistización de lo sacro", la "ética del discurso", el "concepto narrativo del mundo de la vida", la "sistemática de las formas del entendimiento", los tres "medios comunicativos" que son lenguaje, dinero y poder, y la "burocratización de la opinión pública” moderna (Habermas, 2010). Al final, esta concepción de la sociedad "simbólicamente estructurada" se reelabora en función de un planteo "postmetafísico", en el libro titulado, precisamente, 
Pensamiento postmetafísico, a partir de un señalamiento de los "límites de la semántica" y del estudio de la "multiplicidad de voces" de la razón (Habermas, 1990, pp. 113-122; 151 y 182). f) En paralelo, a lo largo de las entrevistas reunidas en Autonomía y solidaridad se elabora la propuesta de una "ética discursiva", especialmente en conexión con la ley y la eticidad (Habermas, 1992, pp. 248-252). Y en Facticidad y validez se introduce la noción de "política deliberativa", en vinculación a las problemáticas de la opinión pública y la democracia (Habermas, 2005, pp. 372-374). g) Finalmente, en Verdad y justificación, puede verse cómo todo lo anterior lleva al autor a reflexionar post-factum sobre el propio "giro lingüístico" como una combinación de hermenéutica y pragmática, con base en una teoría de la verdad de tono "des-trascendental", constituyendo así una mirada sobre la validez “deontológica” de los “juicios” (Habermas, 2002, pp. 65-98 y 261-306).

\section{Metodología: Análisis Crítico del Discurso más Teoría Crítica igual Teoría Crítica del Discurso (ACD+TC=TCD)}

Estos fueron los resultados sintetizados de mi historización conceptual de la teoría crítica. El resultado extenso, detallado y en profundidad de la historización conceptual de la teoría crítica de Horkheimer, Wright Mills y Marcuse puede consultarse en La estructura interna de la teoría crítica (Fraga, 2020). Ahora bien, dados estos resultados, me gustaría pasar ahora a mostrar cómo ellos podrían combinarse con el Análisis Discursivo, y más específicamente con la tradición crítica dentro de él, conocida como Análisis Crítico del Discurso, para finalmente dar forma a mi propuesta particular de una nueva metodología ampliada y específica para la investigación en teoría social: la Teoría Crítica del Discurso.

El análisis crítico del discurso o ACD es la escuela heredera de un escasamente conocido movimiento, dentro de los estudios de análisis discursivo en general, emergido en la década de 1970, con epicentro en la Universidad de East Anglia en Inglaterra, y denominado Lingüística Crítica o LC. Sus referentes principales eran Roger Fowler, Bob Hodge y Gunther Kress, y su hipótesis fundamental era la centralidad de los procesos ocultos de poder producidos y reproducidos en y por el discurso, los cuales, desde su perspectiva, deberían ser no sólo estudiados, sino también modificados en un sentido más igualitario. Esto es claro en los títulos de las obras nodales de tal movimiento: Lenguaje y control(Fowler, Kress, Hodge y Trew, 1979) y Lenguaje como ideología (Kress y Hodge, 1979). La LC nunca tuvo mucho impacto en el resto del campo del análisis discursivo, pero siguió existiendo al punto de que, dos décadas más tarde, ya en 1990, y con epicentro en la Universidad de Amsterdam en Holanda, se conforma un nuevo grupo con los colegas y discípulos de aquella primera camada de investigadores, y que, bajo el nombre de análisis crítico del discurso, sí lograría irrumpir con fuerza en el campo del análisis discursivo en general. Este nuevo grupo se concentra y apoya en torno a la figura del ya para esa época renombrado lingüista neerlandés Teun van Dijk (El prejuicio en el discurso, 1984; Ideología y discurso, 2003), y las figuras principales del ACD propiamente dicho serán el inglés Norman Fairclough y la austríaca Ruth Wodak, todos ellos reunidos además en torno a la novedosa y con el tiempo renombrada publicación periódica Discurso y Sociedad.

Los textos inaugurales del ACD fueron precisamente Lenguaje y poder de Fairclough (1989) y Lenguaje, poder e ideología de Wodak (1989). Aunque la continuidad en los títulos es evidente, el ACD presenta una serie de profundizaciones respecto de su antecesor la LC. En primer lugar, se explicitan por primera vez una serie de perspectivas teóricas de las que estos nuevos estudios se nutrirán: la "crítica ideológica" de Karl Marx (1970); la "sujeción ideológica" de Louis Althusser (1979); los "órdenes del discurso" de Michel Foucault (1997); la "acción comunicativa" de Jürgen Habermas (2010); la "violencia simbólica" de Pierre Bourdieu (1990); el "interaccionismo simbólico" de Herbert Blumer (1986); y la "lingüística sistémico-funcional" de Michael Halliday (1985). En segundo lugar, va tomando forma una lista de temas típicos de estudio a los que quienes adscriben a esta corriente se abocarán: muy especialmente, a las distintas formas de "discriminación", como la "racial", la "étnica" y la de "género", y también a la crítica de diversos formatos discursivos, con énfasis en los "medios de comunicación" como la televisión y el periódico, los discursos orales de "figuras 
públicas" como presidentes o primeros ministros, y las "reglamentaciones institucionales" de espacios clave de la sociedad como los centros educativos o de salud. Así, los principales objetos de crítica de este análisis crítico del discurso son los discursos antisemíticos, machistas (Wodak, 2013), neoliberales y de mercantilización del conocimiento (Fairclough, 1995).

En este marco, comienza quizás a quedar un poco más claro cuál podría ser el aporte de una historia conceptual de la teoría crítica alternativa -esto es, frankfurtiana y nuevaizquierdista- como la que delineé más arriba. En primer lugar, a la serie de perspectivas teóricas de las que la metodología del ACD se nutre -Marx, Althusser, Foucault, Bourdieu, Blumer, Halliday-, creo que puede resultar muy productivo sumar aquellas de Horkheimer, Wright Mills, Marcuse y Gouldner. En efecto, dado que la única perspectiva que ya comparten es la de Habermas, considero que podría ser sumamente fructífero agregar las críticas de la ideología, la metafísica, el cientificismo, del empirismo y el dogmatismo, del sentido común y del saber experto, de los medios de comunicación de masas y de la academia tradicional que realizan los pensadores de la Escuela de Frankfurt y de la Nueva Izquierda. Pero incluso en el caso de Habermas, el ACD utiliza sobre todo sus aportes más clásicos, como aquellos en torno a la acción comunicativa y su distorsión por parte del sistema, por lo que podría ganarse mucho al adicionar aquellos otros, menos conocidos en las ciencias sociales porque han sido apropiados principalmente por la teoría del derecho, acerca de lo que Habermas llama la ética discursiva. En segundo lugar, resulta evidente, a mi modo de ver, la afinidad entre los temas ya estudiados por el ACD y los temas estudiados por estos cinco autores. Por ello, creo que no desmerece sino que complementa al ACD preexistente ampliarle no sólo su espectro teórico, sino también su foco de visión a otros temas, tan relevantes como los primeros en el proceso de develamiento de los procesos de poder en el lenguaje. Estos temas, típicamente analizados por Horkheimer, Wright Mills, Marcuse y Gouldner, aunque también por Habermas, podrían ser: el estudio de los discursos religiosos, filosóficos, psicoanalíticos, artísticos, académicos, partidarios o sindicales; en otras palabras, el estudio de todos aquellos discursos que operan en la conformación de las subjetividades en relación a su sexualidad, su trascendencia, su interioridad, su vocacionalidad, su politicidad, su construcción en términos de identidad o de oposición, y de superioridad o de inferioridad.

En tercer lugar, el pasaje del Análisis Crítico del Discurso a una Teoría Crítica del Discurso vendría dado precisamente por el reemplazo del "análisis crítico" por una "teoría crítica”. El ACD, efectivamente, y aunque se nutre de teorías sociales sobre el discurso, se despliega casi siempre, en las investigaciones efectivas sobre distintos discursos, en la forma de un análisis del contenido y de la forma de distintos textos concretos, de modo similar a como las disciplinas humanísticas interpretan textos arqueológicos, historiográficos, literarios, etc. Este tipo de análisis resulta, a mi modo de ver, una parte fundamental del proceso de observación crítica de la realidad social. Sin embargo, este tipo de análisis empírico deja al margen o pone entre paréntesis la profundización del desarrollo teórico de los propios conceptos o perspectivas utilizados para el análisis discursivo. Así, la TCD sería el momento complementario del ACD: luego de la aplicación de ciertas herramientas teóricas para el análisis discursivo, en el cual ellas son puestas a prueba, dichas herramientas podrían pasar a ser ajustadas, reelaboradas o directamente modificadas o reemplazadas en función de su productividad demostrada -o no- en los análisis concretos de tales o cuales discursos. De este modo, se fortalecería el ida y vuelta entre teoría y empiria, entre textos y herramientas, de modo de robustecer la necesaria circularidad de cualquier emprendimiento de producción de conocimiento sobre el mundo, y muy especialmente de un tipo de conocimiento que busca intervenir sobre ese mundo en un sentido progresista.

\section{Conclusiones: 5 ejes de anÁlisis de una Teoría Crítica del Discurso}

Al final, puedo ahora sí pasar a sistematizar cómo quedaría conformada una Teoría Crítica del Discurso como metodología para la investigación en teoría social. Lo haré a partir de cinco ejes ordenadores, a través de los cuales considero que es factible ubicar las distintas propuestas teóricas de las que tal metodología se 
nutre, combinando tanto los aportes del análisis crítico del discurso con los propios de la historia conceptual de la teoría crítica. Los cinco ejes son: 1) lenguaje en general; 2) discurso ideológico; 3) discurso metafísico; 4) discurso cientificista; y 5) discurso crítico. Cierto que estos 5 ejes podrían reagruparse en 3 grandes grupos. A) En un nivel bien abstracto estarían las conceptualizaciones sobre el lenguaje como fenómeno universal del ser humano (eje 1). B) En un nivel más concreto estarían las conceptualizaciones sobre los distintos tipos de discursos históricos (ejes 2, 3 y 4). C) En un tercer nivel estaría finalmente una reflexión más bien propositiva que analítica, acerca del tipo de discurso que se busca fortalecer en las sociedades actuales (eje 5). Sin embargo, vale la pena ver en detalle qué conceptos específicos podrían aportar cada una de las perspectivas teóricas, tanto las del ACD como las de la teoría crítica, a cada uno de los ejes por separado.

1. Un primer eje es entonces el del análisis del lenguaje en general. Aquí se reúnen las nociones de lo simbólico (Blumer), los símbolos y paleosímbolos (Gouldner), los símbolos-maestros y contra-símbolos (Wright Mills), el concepto de sistema de sentido (Halliday), la diferencia entre sintáctica, semántica y pragmática (Wright Mills), entre habla y escritura, los actos de habla y sus efectos ilocucionarios y perlocucionarios, la acción comunicativa y la racionalidad comunicativa (Habermas).

2. Un segundo eje es el del análisis de los discursos de tipo ideológico. Aquí se reúnen las diversas nociones de ideología entendida como inversión idealista (Marx), como sujeción imaginaria (Althusser), como discurso integrador (Adorno, Horkheimer), como retórica vacía (Wright Mills) o como vocabulario fetichizado (Marcuse). Además, se ubican también aquí los estudios del sentido común emanado y reforzado por los medios de comunicación de masas (Marcuse, Gouldner), por la industria cultural (Adorno, Horkheimer) o aparato cultural (Wright Mills, Althusser).

3. Un tercer eje es el del análisis de los discursos de tipo metafísico. Aquí se reúnen las críticas al pensamiento religioso, sacralizante o fetichizado (Marx), el estudio de los límites a los regímenes de lo decible y lo no-decible (Foucault), al pensamiento rígido anti-dialéctico (Adorno, Horkheimer) y al dogmatismo y la unidimensionalidad de los discursos oficiales (Marcuse).

4. Un cuarto eje es el del análisis de los discursos de tipo cientificista. Aquí se reúnen las críticas a los vocabularios científicos que se presentan como despolitizados (Marx), al campo académicocientífico y su engañoso discurso meritocrático (Bourdieu), y al cientificismo en sus distintas variantes empirista, funcionalista o conductista (Adorno, Horkheimer, Wright Mills, Marcuse, Gouldner).

5. Finalmente, el quinto eje es el del análisis de los discursos de tipo crítico. Aquí se reúnen las propuestas en torno a la crítica (Marx, Althusser, Foucault), a la teoría crítica (Adorno, Horkheimer, Bourdieu), y a la cultura del discurso crítico (Gouldner). Estas incluyen variantes más específicas como el lenguaje artesanal (Wright Mills), la estética crítica (Marcuse) y la ética discursiva (Habermas).

Como puede verse, el primer eje es también un primer paso lógico, pues es preciso tener un entendimiento del funcionamiento del lenguaje como capacidad universal humana para luego proceder a su estudio histórico específico. Luego, los ejes dos, tres y cuatro son a la vez análisis de tipos específicos de lenguaje tanto como críticas a ciertos tipos de discurso, como ser el ideológico, el metafísico y, uno especialmente importante en la época moderna, el científico. Aquí se trata no sólo de entender sino también de evaluar, y entonces logra comenzar a verse el cariz crítico de esta perspectiva metodológica, que arrastra tanto del análisis crítico del discurso como de la teoría social crítica. Finalmente, dicho cariz crítico queda del todo atado a la propuesta metodológica en el quinto eje, que implica el estudio de las posibilidades y las limitaciones del tipo de discurso que los autores de los cuales se nutre buscan promover: el discurso crítico. Se trata entonces así de una 
mirada que no es sólo crítica -de la sociedad y sus discursos- sino también auto-crítica, reflexiva de su propia discursividad.

La elección de estos cuatro tipos de discursos - además del lenguaje en general- no es azarosa ni meramente intuitiva. Existen criterios sistemáticos para dicha distinción, que he desplegado en el libro antes mencionado (Fraga, 2020) -aunque por supuesto, como toda distinción, es de orden analítico, es decir que luego en la práctica los tipos pueden aparecer entremezclados; pero es precisamente parte del trabajo teórico poder "separar los hilos" que en la realidad se encuentran anudados-. Así, por ejemplo, el "lenguaje en general" está obviamente presente en todos los tipos de discursos; del mismo modo, puede decirse, sobre todo desde una perspectiva crítica, que todo lenguaje está impregnado de sujeciones ideológicas así como de compromisos metafísicos; y aún más, que el discurso cientificista es el lenguaje ideológico moderno por antonomasia. Sin embargo, se puede afirmar que los distintos autores de la teoría crítica -en las dos variantes aquí trabajadasdistinguen ellos mismos entre estos distintos tipos de discursos, a los que cuestionan, y a los que a su vez oponen los discursos de tipo crítico, a los cuales defienden. ¿Cuáles son sus características fundamentales, en función de las cuales resulta factible identificarlos?

A partir del estudio de las ideologías más importantes de la modernidad -como el liberalismo o ideología capitalista, con su variante fascista europea y su variante consumista norteamericana, por un lado, y el marxismo ortodoxo o ideología soviética, por otro-, emergen los rasgos fundamentales del discurso ideológico en general. En efecto, se trata de discursos propagandísticos, repetitivos, embotadores, retóricos, competitivos, vulgarizables, mitificadores, folklorizantes, compulsivos, oficializantes, legitimantes, autoritarios, inmunizadores, fetichistas, ritualistas y mecánicos.

A pesar de una preocupación por temas trascendentales, toda vez que el objeto de estudio de las ciencias sociales y humanas es la historia real de las personas y sociedades actuales o potencialmente existentes en este mundo, se intenta dejar claro el límite entre la utopía como motor del pensamiento y la pura metafísica. Así, el discurso metafísico es aquel que resalta por su dogmatismo, oscurantismo, esencialismo, sacralismo, fundamentalismo, universalismo, objetivismo, naturalismo, así como por su carácter sublimante, ocultador, reificado, determinista, fatalista e incluso a veces totalitario.

Un discurso particularmente penetrante en la modernidad es el cientificista, es decir, aquel que sacraliza la ciencia, típico de las sociedades capitalistas, industrialistas, tecnológicas y/o de consumo. El estudio del cientificismo permite presentarlo como un discurso tecnocrático, empresarial, burocratizante, tecnicista, experto, estratégico, conductista, amoral, operacionalista, empirista, positivista, sobresimplificador y antihumanista, que además mantiene vínculos con otros discursos como el nacionalismo, el belicismo, la justificación de lo estatuido y la carencia de reflexión sobre sus fines últimos.

Finalmente, los discursos cientificistas, ideológicos y metafísicos contrastan con otro tipo de discurso, el que es paradigmático de la teoría crítica. El discurso crítico, en efecto, presenta objetos y objetivos, métodos e intervenciones, tradiciones y deseos muy distintos respecto a los anteriores. El discurso crítico implica tomar distancia de -y derribar- los presupuestos de los discursos metafísicos, ideológicos y cientificistas, así como un corte con el pensamiento y el lenguaje de sentido común y con la opinión corriente. La teoría crítica es una teoría histórica, materialista, dialéctica, reflexiva, normativa, política, denunciadora, oposicionista, controversial, creativa, transformadora, constructiva y humanista.

Con todo, puede quedar aún una duda, que refiere a la diversidad de compromisos epistemológicos que sustentan los distintos autores de una Teoría Crítica del Discurso. En efecto, algunos de ellos presentan posiciones más universalistas y otros más particularistas; unos presentan posturas más realistas y otros más constructivistas, etc. Sin embargo, ya la perspectiva del Análisis Crítico del Discurso, asentada y aceptada como legítima hace tiempo, comparte esta misma apertura a la variedad de puntos de vista, a la que no sólo tolera sino que la incentiva, pues considera que es precisamente esa variedad la que enriquece el paradigma y las herramientas. Luego, dentro del amplio paraguas que queda constituido con tal variedad, cada investigador o grupo de investigadores puede optar por tomar una, algunas o todas las perspectivas 
teóricas ofrecidas y sistematizadas. Así, por ejemplo, el Abordaje Histórico-Discursivo de Wodak utiliza primariamente a Adorno y Blumer; el Abordaje Dialéctico-Relacional de Fairclough hace uso sobre todo de Marx, Foucault y Halliday; y el Abordaje Socio-Cognitivo de van Dijk se apoya en la psicología social de Serge Moscovici. Finalmente, otras escuelas dentro del ACD declinan sus investigaciones con los estudios de Mikhail Bajtin, Paul H. Grice o Aron Cicourel.

Entonces ahora sí, con estos cinco ejes, y estas aclaraciones y propuestas, queda constituido el esqueleto de la metodología de la Teoría Crítica del Discurso, la cual, esperamos, podrá ser llenada y puesta a prueba en el futuro con la carne de los análisis discursivos concretos.

\section{REFERENCIAS}

Abend, G. (2008). The meaning of theory. Sociological Theory, 26(2), 173-199.

Adorno, T. (2008). Crítica de la cultura y sociedad I y II. Madrid: Akal.

Aguilar, P. L., Glozman, M., Grondona, A. L. y Haidar, V. (2014). ¿Qué es un corpus? Entramados yperspectivas, 4(4), 35-64.

Althusser, L. (1979). Ideología y aparatos ideológicos de estado. En Posiciones. Barcelona: Anagrama.

Álvarez Ruiz, F. (2019). Hacia una crítica de la totalidad eurocéntrica como fundamento para estudios de teoría social de (y desde) el sur. (pp. 29-68). En P. de Marinis (coord.), Exploraciones en teoría social. Ensayos de imaginación metodológica. Buenos Aires: IIGG-CLACSO.

Alvaro, D. (2017). Sociología y deconstrucción. Cuestiones de método. Cuadernos de Teoría Social, 3(5), 8-27.

Alvear, R. (2015). El lenguaje como herramienta para la configuración de una teoría crítica de la sociedad. Un acercamiento a la teoría de Habermas. Persona y Sociedad, 29(1), 85-111.

Aune, J. A. (1990). Cultures of discourse. Marxism and rhetorical theory. En D. C. Williams y M. D. Hazen (eds.), Argumentation theory and the rhetoric of assent (p. 155-176).. Tuscaloosa: University of Alabama.

Barbieri Durao, A. (2015). A teoria do discurso racional de Habermas. Filosofia moderna e contemporanea, 3(1), 6-27.

Benhabib, S. (1986). Critique, norm and utopia. A study of the foundations of critical theory. Nueva York: Columbia University.

Bialakowsky, A. (2013). Antecedentes y posibilidades de un análisis comparativo en metateoría. El abordaje problemático en la teoría sociológica contemporánea. Documentos de Jóvenes Investigadores, 38, 1-60.

Blumenberg, H. (2003). Paradigmas para una metaforologia. Madrid: Trotta.

Blumer, H. (1986). Symbolic interactionism. Perspective and method. Los Ángeles: University of California.

Bourdieu, P. (1990). Sociología y cultura. México: Grijalbo.

Brígido, E. I. (2017). Habermas e a ética do discurso. Saberes, 1(15), 134-150.

Casagrande, C. A. y Casagrande, E. F. (2011). A ética discursiva e o caráter procedimental do discurso prático em Habermas. Conjectura, 16(3), 131-145.

Castel, R. (2007). Presente y genealogía del presente. Una aproximación no evolucionista al cambio histórico. Au risque du Foucault. (pp. 161-168). París: Centre Pompidou.

Cristiano, J., Torres, E., Gonnet, J. P. y Abril, F. (2015). Operaciones y procedimientos de la construcción teórica. Presentación preliminar de un instrumento de análisis. Trabajo presentado en I Congreso Latinoamericano de Teoría Social. Buenos Aires, Argentina.

Crook, S. (1991). Horkheimer's 'critical theory'. Modernist radicalism and its aftermath. Foundationalism and antifoundationalism in radical social theory. (pp. 87-92). Londres: Routledge.

Cukier, W., Bauer, R. y Middleton, C. (2003). Applying Habermas' validity claims as a standard for critical discourse analysis. (pp. 233-258). En B. Kaplan, D. Truex, T. Wood Harper, y J. De Gross (eds.), Information systems research. Relevant theory and informed practice. Dordrecht: Kluwer.

Daley, P. (1991). Alvin Gouldner's views on the public sphere. Journal of Communication Inquiry, 15(2), 107-125. 
De Marinis, P. (2013). Gemeinschaft, community, comunidad: algunas reflexiones preliminares acerca de las variadas semánticas de la comunidad en la teoría sociológica. Revista Argentina de Ciencia Política, 16, 87-104.

Diniz Focas, J. (2016). Habermas e Ducrot. Percursos da ética de discurso. Estudos Integrados em Discurso e Argumentacao, 12, 119-134.

Ducrot, O. (1990). Polifonia y argumentación. Cali: Universidad del Valle.

Fairclough, N. (1989). Language and power. Londres: Longman.

Fairclough, N. (1995). Critical discourse analysis. Boston: Addison Wesley.

Fernández Cordero, M. L. (2013). Un ejercicio de lectura sobre el concierto de la prensa anarquista a partir de Mijail Bajtin (Argentina, 1895-1925). AdVersus, 10(24), 68-91.

Fernández Sergio, P. (1997). Habermas y la Teoría Crítica de la Sociedad. Legado y diferencias en teoría de la comunicación. Cinta de Moebio, 1, 27-41.

Foucault, M. (1997) [1966]. Laspalabras y las cosas. Una arqueología de las ciencias humanas. Buenos Aires: Siglo XXI. Fowler, R., Kress, G., Hodge, R. y Trew, T. (1979). Language and control. Londres: Routledge.

Fraga, E. (2014). ¡El futuro será de los artistas! Un análisis discursivo del manifiesto expresionista. Revista Athenea, 14(2), 39-69.

Fraga, E. (2015a). Dialógica, polémica, retórica, tópica y deíctica. Las dimensiones argumentativas de la crítica. Revista Horizontes Filosóficos, 5, 39-60.

Fraga, E. (2015b). La comunidad en Walter Mignolo. Cinco dimensiones de un mismo concepto. Revista e-l@tina, $13(51), 1-15$.

Fraga, E. (2017). Teoría sociológica y teoría critica. Un diálogo posible entre dos proyectos (no tan) contrapuestos. Universidad de Buenos Aires.

Fraga, E. (2018a). La disputa epistemológica contra el empirismo y la propuesta de la teorización sistemática. Revista Cinta de Moebio, 61, 28-40.

Fraga, E. (2018b). Formas de teorizar en ciencias sociales en Argentina y Latinoamérica. Revista Papeles de Trabajo, 12(22), 192-203.

Fraga, E. (2019a). Sociología radical y Nueva izquierda. Elementos para una teoría crítica alternativa. Universidad de Buenos Aires.

Fraga, E. (2019b). ¿Qué es, cómo se hace y para qué sirve la teoría? Aportes desde la sociología y desde sus márgenes. Revista CS del ICESI, 28, 181-206.

Fraga, E. (2020), La estructura interna de la teoría critica: Horkheimer, Wright Mills, Marcuse. Buenos Aires: CAL.

Fraser, N. (1997). Iustitia interrupta. Reflexiones criticas desde una posición postsocialista. Colombia: Siglo del Hombre.

Gadamer, H. G. (1997). Historia y hermenéutica. Barcelona: Universidad Autónoma de Barcelona.

Gil Martín, F. J. (2005). La razón práctica y el principio del discurso en Jürgen Habermas. Revista de Filosofía, 23(50), $1-25$.

Girola, L. (2011). Historicidad y temporalidad de los conceptos sociológicos. Sociológica, 73(26), 13-46.

Gouldner, A. W. (1973) [1970]. La crisis de la sociología occidental. Buenos Aires: Amorrortu.

Gouldner, A. W. (1978) [1976]. La dialéctica de la ideología y la tecnología. Los origenes, la gramática y el futuro de la ideologia. Madrid: Alianza.

Gouldner, A. W. (1979) [1973]. La sociología actual: renovación y critica. Madrid: Alianza.

Gouldner, A. W. (1980) [1979]. El futuro de los intelectuales y el ascenso de la nueva clase. Madrid: Alianza.

Gouldner, A. W. (1983) [1980]. Los dos marxismos: contradicciones y anomalias en el desarrollo de la teoría. Madrid: Alianza.

Gouldner, A. W. (1985). Against fragmentation. The origins of marxism and the sociology of intellectuals. Nueva York: Oxford University.

Gramsci, A. (1984) [1949]. Los intelectuales y la organización de la cultura. Buenos Aires: Nueva Visión. 
Habermas, J. (1981) [1962]. Historia y crítica de la opinión pública. Barcelona: Gustavo Gili.

Habermas, J. (1982) [1968]. Conocimiento e interés. Madrid: Taurus.

Habermas, J. (1987) [1967]. La lógica de las ciencias sociales. Madrid: Tecnos.

Habermas, J. (1990) [1988]. Pensamento pós-metafísico. Río de Janeiro: Tempo Brasileiro.

Habermas, J. (1992) [1986]. Autonomy and solidarity. Londres: Verso.

Habermas, J. (1998) [1976-1985]. On the pragmatics of communication. Massachussetts: MIT Press.

Habermas, J. (2001) [1971-1974]. On the pragmatics of social interaction. Preliminary studies in the theory of communicative action. Massachussetts: MIT Press.

Habermas, J. (2002) [1999]. Verdad y justificación. Madrid: Trotta.

Habermas, J. (2005) [1992]. Facticidad y validez. Sobre el derecho y el estado democrático de derecho en términos de teoria del discurso. Madrid: Trotta.

Habermas, J. (2008) [1985]. El discurso filosófico de la modernidad. Buenos Aires: Katz.

Habermas, J. (2010) [1981]. Teoria de la acción comunicativa Iy II. Madrid: Trotta.

Halliday, M. A. K. (1985). An introduction to functional grammar. Londres: Baltimore.

Heidegger, M. (2012) [1927]. Ser y tiempo. Madrid: Trotta.

Herrera Gómez, M. (2003). Lenguaje y acción en la teoría de la acción comunicativa de Habermas. Revista de Estudios Politicos, 121, 31-70.

Honneth, A. (1997). La lucha por el reconocimiento. Por una gramática moral de los conflictos sociales. Barcelona: Crítica.

Horkheimer, M. (1973) [1946]. Critica de la razón instrumental. Buenos Aires: Sur.

Horkheimer, M. (1974) [1932-1941]. Teoria critica. Buenos Aires: Amorrortu.

Horkheimer, M. (1995) [1930-1938]. Historia, metafisica y escepticismo. Barcelona: Altaya.

Horkheimer, M. (2002) [1974]. Ocaso. Madrid: Editora Nacional.

Horkheimer, M. y Adorno, T. (2001) [1949]. Dialéctica de la ilustración. Fragmentos filosóficos. Madrid: Trotta.

Horowitz, I. L. (1970). Estilo y contenido de Mills. En C. Wright Mills, De hombres sociales y movimientos politicos. (pp. 11-19). México: Siglo XXI.

Jaeggi, R. (2018). Critique of forms of life. Cambridge: Harvard University.

Korsch, K. (1971) [1923]. Marxismo y filosofia. Madrid: Ariel.

Koselleck, R. (2012). Historias de conceptos. Estudios sobre semántica y pragmática del lenguaje politico y social. Madrid: Trotta.

Kress, G. y Hodge, R. (1979). Language as ideology. Londres: Routledge.

Labandeira, M. C. (2008). Materialismo del encuentro. Una filosofía para la teoría del discurso. AdVersus, 5(12), 36-79.

Loy, J. y Booth, D. (2004). Consciousness, craft, commitment. The sociological imagination of C. Wright Mills. En R. Giulianotti (comp.), Sport and modern social theorists. Hampshire: Palgrave Macmillan.

Lubenow, J. A. (2011). Sobre o método do discurso prático na fundamentacao da ética do discurso de Habermas. Cadernos do PET Filosofia, 2(3), 57-70.

Lukács, G. (2013) [1923]. Historia y conciencia de clase. Buenos Aires: Razón y revolución.

Marcuse, H. (1972). Counterrevolution and revolt. Boston: Beacon.

Marcuse, H. (1978). The aesthetic dimension. Toward a critique of marxist aesthetics. Boston: Beacon.

Marcuse, H. (1984) [1958]. El marxismo soviético. Madrid: Alianza.

Marcuse, H. (1985) [1964]. El hombre unidimensional. Ensayo sobre la ideología de la sociedad industrial avanzada. Barcelona: Planeta-Agostini.

Marx, K. y Engels, F. (1970) [1845]. La ideología alemana. Barcelona: Grijalbo.

Mascareño, A. (2006). Sociología del método: La forma de la investigación sistémica. Cinta de Moebio, 26, 122-154.

Mead, G. H. (1934). Mind, self and society. Chicago: Chicago University Press. 
Montero, A. S. (2012). Los usos del ethos: abordajes discursivos, sociológicos y políticos. Rétor, 2(2), 223-242.

Palma, A. G. (2011). Las concepciones del lenguaje en Gadamer y Habermas. Hacia posibles aportes para un análisis crítico del discurso. Cuadernos del Sur, 40, 133-152.

Palti, E. (2005). De la historia de las ideas a la historia de los lenguajes políticos. Las escuelas recientes de análisis conceptual: el panorama latinoamericano. Anales, 8, 63-82.

Pêcheux, M. (2016). Las verdades evidentes. Lingüistica, semántica, filosofía. Buenos Aires: Ediciones CCC. Peirce,

C. S. (1934). Collected Papers Vols. I-V. Massachussetts: Cambridge.

Pels, D. (2001). Three spaces of social theory. Towards a political geography of knowledge.Canadian Journal of Sociology. 26(1), 31-56.

Persch, D. (2009). A ética do discurso de Habermas. Fundamentacao e aplicabilidade. Filosofazer, 35, 69-87.

Pignuoli Ocampo, S. (2013). Reflexiones sobre la metateoría y los problemas metodológicos fundamentales de la teoría sociológica comparada. Presentado en X Jornadas de Sociología de la Universidad de Buenos Aires, Buenos Aires.

Repa, L. (2008). A normatividade do discurso em Habermas. Doispontos, 5(2), 89-111.

Requena Santos, F. (2000). Hacia una perspectiva reticular de la teoría sociológica. Papers, 62, 133-145.

Ritzer, G. (1990). Metatheorizing in sociology. Sociological Forum, 5(1), 3-15.

Rosa, H. (2015). Social acceleration. Nueva York: Columbia University.

Sasín, M. G. (2019). Investigación teórica, semántica y comunicación. El lugar de lo masivo en la teoría de los sistemas sociales autopoiéticos (Un ejercicio de aplicación). (pp. 305-340) En P. de Marinis (coord.), Exploraciones en teoría social. Ensayos de imaginación metodológica. Buenos Aires: IIGG-CLACSO.

Saussure, F. (1997). Curso de lingüistica general. Buenos Aires: Losada.

Schluchter, W. (2008). Acción, orden y cultura. Estudios para un programa de investigación en conexión con Max Weber. Buenos Aires: Prometeo.

Schmidt, J. (1998). Language, mythology and enlightenment. Historical notes on Horkheimer and Adorno's Dialectic of enlightenment. Social Research, 65(4), 807-838.

Skinner, Q. (2000). Significado y comprensión en la historia de las ideas. Prismas, 4, 149-191.

Slater, I. (1975). Orwell, Marcuse, and the language of politics. Political Studies, 23(4), 459-474.

Soares Teixeira, M. (2016). Ética do discurso en Habermas. A importancia da linguagem. Opinao Filosófica, 7(2), 304-315.

Somers, M. (1995). What's political or cultural about the political culture concept? Toward an historical sociology of concept formation. Sociological Theory, 13(2), 113-144.

Torres Oviedo, J. M., Galván Patrignani, G. y Hernández Palma, O. I. (2016). Ética del discurso de Habermas. Propuesta teórico-práctica para la solución de problemas práctico-morales. Justicia, 29, 13-27.

Trovero, J. I. (2019). De la teoría a la teorización. Algunos aportes para el trabajo en/con teoría en sociología. (pp. 379-410). En P. de Marinis (coord.), Exploraciones en teoría social. Ensayos de imaginación metodológica. Buenos Aires: IIGG-CLACSO.

Van Dijk, T. (1984). Prejudice in discourse. Amsterdam: Benjamins.

Van Dijk, T. A. (2003). Ideología y discurso. Una introducción multidisciplinaria. Barcelona: Ariel.

Wright Mills, C. (1961) [1959]. La imaginación sociológica. México: Fondo de Cultura Económica.

Wright Mills, C. (1963) [1939-1960]. Power, politics and people. The collected essays. Nueva York: Ballantine.

Wright Mills, C. (1968) [1941]. Sociología y pragmatismo. Buenos Aires: Siglo Veinte.

Wright Mills, C. (1970) [1939-1960]. De hombres sociales y movimientos politicos. México: Siglo XXI.

Wright Mills, C. (1976) [1962]. Los marxistas. México: Era.

Wright Mills, C. (2000). Letters and autobiographical writings. Berkeley: University of California.

Wright Mills, C. y Gerth, H. (1961) [1953]. Character and social structure. Londres: Routledge \& Kegan Paul.

Wodak, R. (1989). Language, power and ideology. Amsterdam: Benjamins. 
Wodak, R. (2013). Critical discourse analysis. Vol. 1: Concepts, history, theory. Londres: Sage.

Zabludovsky, G. (2002). Teoría y metateoría en las ciencias sociales contemporáneas. (pp. 127-168). En Sociología y política, el debate clásico y contemporáneo. México: Porrúa. 